\title{
Smartphone Addiction Proneness Is Associated With Subjective-Objective Sleep Discrepancy in Patients With Insomnia Disorder
}

\author{
Jeewon Lee, Han-Yong Jung, Soyoung Irene Lee, HyunChul Youn, and Shin-Gyeom Kim ${ }^{凶}$ \\ Department of Psychiatry, Soonchunhyang University College of Medicine, Bucheon Hospital, Bucheon, Republic of Korea
}

Objective Subjective reports of patients with insomnia often show a discrepancy with their objective assessments of sleep. We aimed to assess subjective-objective sleep discrepancy in subjects with insomnia disorder as well as the psychological factors associated with the discrepancy.

Methods This study is a secondary analysis of the baseline data of a randomized controlled study on 110 adults aged 18 years to 59 years with insomnia disorder. Subjective reports on sleep and the objective measures acquired by an overnight polysomnography were used to measure the sleep discrepancy. Smartphone Addiction Proneness Scale (SAPS), Center for Epidemiologic Studies Depression Scale (CES-D), beck anxiety inventory (BAI), and Global Assessment of Recent Stress (GARS) were used to evaluate the psychological factors associated with the sleep discrepancy.

Results Mean total sleep time (TST) discrepancy of the participants was $-81.65 \pm 97.41$ minutes. Multivariable logistic regression analyses revealed that age (adjusted $\mathrm{OR}=1.07,95 \% \mathrm{CI}=1.01-1.13, \mathrm{p}=0.027$ ), years of education (adjusted $\mathrm{OR}=0.69,95 \% \mathrm{CI}=0.48-0.91$, $\mathrm{p}=0.017$ ), and smartphone addiction proneness (adjusted $\mathrm{OR}=1.14,95 \% \mathrm{CI}=1.04-1.27, \mathrm{p}=0.008$ ) were independent predictors of TST misperception. Mean sleep onset latency (SOL) discrepancy of the participants was $41.28 \pm 45.01$ minutes. Only anxiety was an independent predictor of SOL misperception (adjusted OR=1.16, 95\% CI=1.05-1.31, $\mathrm{p}=0.006$ ).

Conclusion The present study provides empirical evidence to increase our understanding of the various factors that are associated with subjective-objective sleep discrepancy. Screening insomnia patients with smartphone addiction proneness may help predict the potential discrepancy between the patients' subjective reports and objective measures of sleep duration.

Psychiatry Investig 2021;18(11):1035-1043

Keywords Insomnia; Sleep discrepancy; Sleep misperception; Smartphone addiction.

\section{INTRODUCTION}

Insomnia is a highly prevalent clinical complaint that affects $10 \%-50 \%$ of the general population. ${ }^{1-4}$ The prevalence of insomnia has been continuously increased over the past decade in Korea. ${ }^{5,6}$ Clinical diagnosis of insomnia disorder relies on subjective reports of difficulty initiating or maintaining sleep, or early morning awakening which causes functional impairment. ${ }^{7}$ Objective measurements of sleep obtained by

Received: September 28, 2020 Revised: April 11, 2021

Accepted: June 9, 2021

$\square$ Correspondence: Shin-Gyeom Kim, MD, PhD

Department of Psychiatry, Soonchunhyang University College of Medicine, Bucheon Hospital, 170 Jomaru-ro, Bucheon 14584, Republic of Korea

Tel: +82-32-621-5063, Fax: +82-32-621-6950, E-mail: redmensch@schmc.ac.kr

(c) This is an Open Access article distributed under the terms of the Creative Commons Attribution Non-Commercial License (https://creativecommons.org/licenses/by$\mathrm{nc} / 4.0$ ) which permits unrestricted non-commercial use, distribution, and reproduction in any medium, provided the original work is properly cited. polysomnography or actigraphy are not regarded as prerequisites for the diagnosis of insomnia disorder in clinical practice. However, it has been reported that subjective reports of patients with insomnia often show a discrepancy with their objective assessments of sleep. Insomnia patients have a tendency to underestimate their total sleep time (TST) or overestimate their sleep onset latency (SOL), or both. ${ }^{8}$ Such difference between subjective and objective sleep parameters is called subjective-objective sleep discrepancy. ${ }^{9}$

Subjective-objective sleep discrepancy is also referred to as sleep misperception..$^{10}$ Some extent of subjective-objective sleep discrepancy is known as a ubiquitous clinical feature of individuals with insomnia. ${ }^{8}$ The sleep discrepancy should be an important consideration when assessing and treating insomnia patients in clinical practice. Subjects with significant sleep discrepancy may not respond to treatment as others do, and they may need a different kind of treatment approach. ${ }^{11}$ Fur- 
thermore, sleep discrepancy can perpetuate chronic insomnia. ${ }^{10}$ Those who have a negatively distorted cognition on their sleep may be at a greater risk of developing more severe insomnia symptoms due to increased preoccupation with sleep and increased sleep related anxiety. ${ }^{12}$

Little is known about the underlying mechanisms of sleep discrepancy. Many psychological, cognitive, and physiological factors have been proposed as contributing factors to the phenomenon. For example, cognitive arousal, sleep effort, mood upon awakening, and fragmentation of sleep architecture have been proposed as predicting factors of sleep misperception. ${ }^{13}$ Also, depressive, anxious-ruminative personality traits and poor stress-coping resources have been reported to be associated with sleep misperception. ${ }^{14}$ Additionally, memory bias and cognitive distortion have been suggested as an explanation for the subjective-objective sleep discrepancy. ${ }^{10,12,15}$

Most recently, smartphones have become a necessity for our lives. A previous study conducted in 2015 showed that young adults use their smartphone for more than 5 hours daily and check their smartphone more than 60 times a day. ${ }^{16}$ While the innovate technology provides so much convenience to all the users, excessive and uncontrolled smartphone use has yielded various negative consequences. Particularly regarding sleep, bedtime smartphone use can have detrimental effects on sleep and daytime function in adults. ${ }^{17}$ Fossum et al. ${ }^{18}$ have reported that use of mobile phone for texting, playing, and surfing in bed before going to sleep is positively associated with insomnia symptoms. Also, mobile phone usage in bed was significantly associated with feeling less refreshed and sleepier during the day. ${ }^{19}$ Those who use their smartphone before going to sleep more often had the impression of not getting enough sleep.

There is substantial evidence from the previous studies that excessive smartphone usage is associated with negative sleep quality. However, no previous study has evaluated whether problematic smartphone usage itself could predict one's tendency to misperceive one's sleep problem. Many factors that have been shown to be associated with sleep discrepancy were factors that cause sleep problems. ${ }^{13,14}$ Therefore, we hypothesized that smartphone addiction proneness may also predict sleep discrepancy.

In the present study, we aimed to examine the subjectiveobjective sleep discrepancy in adults with insomnia disorder. We assessed various psychological factors that could be associated with the sleep misperception that underestimates the total sleep time and overestimates the sleep onset latency. To our knowledge, we present the first study results showing the association between smartphone addiction proneness and sleep discrepancy in insomnia patients.

\section{METHODS}

Data for this study were drawn from a clinical study on adults with insomnia disorder. ${ }^{20}$ Baseline measurements of the participants were analyzed to examine the characteristics of subjects according to their sleep discrepancy. This study was approved by the Institutional Review Board of the Soonchunhyang University Bucheon Hospital (SCHBC 2017-029-022).

\section{Participants}

The inclusion criteria of the participants were adults aged 18 to 59 years who met the diagnostic criteria of insomnia disorder according to the Diagnostic and Statistical Manual 5 criteria. ${ }^{7}$ Exclusion criteria included major psychiatric disorders (schizophrenia, major depressive disorder, bipolar disorder, substance use disorder), developmental disorders (intellectual disability and autism spectrum disorder), neurocognitive disorders (delirium and dementia), neurologic disorders (epilepsy and cerebrovascular disease), any ongoing severe medical condition, history of suicidal attempt, consumption of any kind of sleep medications during the past month, and currently a shift-worker. Participants with loud snoring or who had been diagnosed with OSA and those with body mass index $(\mathrm{BMI})>30 \mathrm{~kg} / \mathrm{m}^{2}$ were also excluded.

Participants were recruited through advertisements and clinical referrals from November 2017 to December 2018. The advertisement stated that we were recruiting subjects for the double-blind, randomized, placebo-controlled study which was for the assessment of the efficacy and safety of passionflower extracts in adults with insomnia. All advertisements were approved by the Institutional Review Board. Written informed consent was obtained from each subject prior to participation. Among the 110 participants enrolled in the clinical study, 105 participants who had undergone baseline polysomnography tests and had a score above 5 on pittsburgh sleep quality index (PSQI) were included in the analysis for the present study.

\section{Measurements}

\section{Subjective reports on sleep}

Insomnia severity index (ISI) is a brief self-report questionnaire with 7 items to assess severity of both nighttime and daytime symptoms of insomnia during the past 2 weeks. ${ }^{21}$ Total score ranges from 0-28: 0-7 no clinically significant insomnia; 8-14 subthreshold insomnia; 15-21 clinical insomnia (moderate severity); 22-28 clinical insomnia (severe).

PSQI is a 19-item self-report questionnaire that assesses the sleep quality over the past month on a $0-3$ scale. ${ }^{22}$ It creates 7 components (subjective sleep quality, sleep latency, sleep du- 
ration, habitual sleep efficiency, sleep disturbances, use of sleeping medication, and daytime dysfunction) and 1 global score that ranges from 0 to 21 . A cut-off score of 5 is used to identify cases with sleep disorder.

Epworth Sleepiness Scale (ESS) measures the general level of daytime sleepiness. ${ }^{23}$ It consists of 8 situations in which one rate one's chance of dozing from $0-3$ scale. Total score ranges from 0 to 24 and scores higher than 10 may indicate excessive sleepiness which needs medical attention.

Sleep duration and sleep latency reported on PSQI were used as subjective reports of total sleep time (sTST) and sleep onset latency (sSOL).

\section{Polysomnographic sleep parameters}

Participants underwent an overnight polysomnography. All participants were monitored in a supervised sleep laboratory overnight. The lights off time was 10:30 pm. Participants were awakened the next morning at 06:30 am. Objective sleep parameters including total sleep time (oTST), sleep efficiency (SE), sleep onset latency (oSOL), wake after sleep onset (WASO), number of total arousal, and more were obtained by the polysomnography.

\section{Psychological measures}

Smartphone Addiction Proneness Scale (SAPS) was developed by the Korean National Information Society Agency for screening subjects who may be at risk of smartphone addiction. ${ }^{24}$ It consists of 15 items with 4 domains: daily life disturbance (5 items), virtual life orientation ( 2 items), withdrawal ( 4 items), and tolerance ( 4 items). These items are scored on a 4-point Likert scale and higher scores indicate higher risk of smartphone addiction.

Center for Epidemiologic Studies Depression Scale (CES-D) is a screening instrument for depression. ${ }^{25}$ It consists of 20 items with 4-point Likert scale. A score of 15 or higher is interpreted to indicate a risk for depression.

Beck anxiety inventory (BAI) is a self-report inventory that measure the severity of anxiety during the past week. ${ }^{26} \mathrm{BAI}$ consists of 21 items with 4-point Likert scale. A score of 10 or higher is interpreted to indicate risk for anxiety disorder.

Global Assessment of Recent Stress (GARS) was developed to assess the current level of perceived stress during the previous week. ${ }^{27}$ It assesses 7 areas including work pressure, interpersonal relationship, changes in relationships, sickness or injury, financial issues, unusual happenings, change or lack of change from routine.

\section{Categorization of sleep misperception groups}

Sleep discrepancy value for TST was calculated by subtracting the subjective TST from the objective TST. Participants
Table 1. Demographic and clinical characteristics of the participants $(\mathrm{N}=105)$

\begin{tabular}{|c|c|}
\hline Variables & Mean \pm SD, N (\%) \\
\hline Age, years & $40.61 \pm 11.62$ \\
\hline Female & $49(46.7)$ \\
\hline Education, years & $15.08 \pm 2.21$ \\
\hline \multicolumn{2}{|l|}{ Subjective sleep reports } \\
\hline sTST, min & $316.69 \pm 70.57$ \\
\hline sSOL, min & $57.45 \pm 41.60$ \\
\hline ISI & $14.47 \pm 4.23$ \\
\hline PSQI & $8.64 \pm 1.49$ \\
\hline ESS & $9.25 \pm 4.27$ \\
\hline \multicolumn{2}{|l|}{ Polysomnographic sleep parameters } \\
\hline oTST, min & $398.33 \pm 62.13$ \\
\hline SE, \% & $83.18 \pm 13.35$ \\
\hline oSOL, min & $16.17 \pm 24.51$ \\
\hline REM latency, min & $119.43 \pm 69.45$ \\
\hline N1, \% & $13.59 \pm 9.65$ \\
\hline $\mathrm{N} 2, \%$ & $63.54 \pm 9.17$ \\
\hline $\mathrm{N} 3, \%$ & $4.20 \pm 5.99$ \\
\hline NREM, \% & $81.33 \pm 5.88$ \\
\hline REM, \% & $18.67 \pm 5.88$ \\
\hline WASO, min & $64.62 \pm 57.67$ \\
\hline Total arousal, $\mathrm{N}$ & $118.85 \pm 68.87$ \\
\hline $\mathrm{AHI}$ & $7.26 \pm 11.91$ \\
\hline \multicolumn{2}{|l|}{ Psychological measures } \\
\hline CES-D & $12.47 \pm 9.59$ \\
\hline BAI & $9.78 \pm 8.31$ \\
\hline GARS & $23.94 \pm 12.25$ \\
\hline SAPS & $29.64 \pm 6.18$ \\
\hline \multicolumn{2}{|l|}{ Sleep discrepancy } \\
\hline Mean $\Delta$ TST (sTST-oTST), min & $-81.65 \pm 97.41$ \\
\hline TST Misperception $(\Delta \mathrm{TST}<-60)$ & $65(61.90)$ \\
\hline $\begin{array}{l}\text { No TST Misperception } \\
(-60 \leq \Delta \mathrm{TST} \leq 60)\end{array}$ & $33(31.43)$ \\
\hline Excluded $(\Delta \mathrm{TST}>60)$ & $7(6.67)$ \\
\hline Mean $\Delta$ SOL (sSOL-oSOL), min & $41.28 \pm 45.01$ \\
\hline SOL Misperception $(\Delta \mathrm{SOL}>30)$ & $55(52.38)$ \\
\hline $\begin{array}{l}\text { No SOL Misperception } \\
(30 \geq \Delta \mathrm{SOL} \geq-30)\end{array}$ & $49(46.67)$ \\
\hline Excluded $(\Delta \mathrm{SOL}<-30)$ & $1(0.95)$ \\
\hline
\end{tabular}

TST, total sleep time; SOL, sleep onset latency; ISI, insomnia severity index; PSQI, pittsburgh sleep quality index; ESS, Epworth Sleepiness Scale; SE, sleep efficiency; WASO, wake after sleep onset; AHI, apnea-hypopnea index; CES-D, Center for Epidemiologic Studies Depression Scale; BAI, beck anxiety inventory; GARS, Global Assessment of Recent Stress Scale; SAPS, Smartphone Addiction Proneness Scale 
who had TST discrepancy smaller than -60 minutes, which means they have underestimated their TSTs for more than 60 minutes, were categorized as TST Misperception group. This is in line with many previous studies that used the value of 60 minutes of TST underestimation for the clinically reasonable cut-off point for separating those with or without sleep misperception. ${ }^{14,28-32}$ Those with TST discrepancy between 60 minutes to -60 minutes were categorized as No TST Misperception group. Those with TST discrepancy larger than 60 minutes, which means they have overestimated their TSTs for more than 60 minutes, were excluded from the analysis.

Sleep discrepancy value for SOL was calculated by subtracting the subjective SOL from the objective SOL. Participants who had a SOL discrepancy larger than 30 minutes, which means they have overestimated their SOLs for more than 30 minutes, were categorized as SOL Misperception group. A theoretical review on sleep misperception had noted that the estimation error between subjective and objective SOL for the diagnosis of sleep misperception should not be less than 29 minutes. ${ }^{33}$ Those with a SOL discrepancy between 30 minutes to - 30 minutes were categorized as No SOL Misperception group. Those with a SOL discrepancy less than -30 minutes, which means they have underestimated their SOLs for more than 30 minutes, were excluded from the analysis.

\section{Statistical analysis}

Group differences on the demographic and clinical variables were analyzed using independent t-tests or Mann-Whit-

Table 2. Characteristics of the participants with or without TST misperception

\begin{tabular}{|c|c|c|c|}
\hline Variables & TST misperception $(\mathrm{N}=65)$ & No TST misperception $(\mathrm{N}=33)$ & $\mathrm{p}$ \\
\hline Age, years & $41.85 \pm 11.88$ & $38.85 \pm 10.76$ & 0.229 \\
\hline Gender, Female & $36(55.38)$ & $17(51.52)$ & 0.882 \\
\hline Education, years & $14.74 \pm 2.16$ & $15.91 \pm 2.16$ & 0.036 \\
\hline \multicolumn{4}{|l|}{ Subjective sleep reports } \\
\hline sTST, min & $280.95 \pm 50.07$ & $366.06 \pm 56.73$ & $<0.001^{* * *}$ \\
\hline sSOL, min & $52.58 \pm 47.37$ & $58.97 \pm 38.83$ & 0.123 \\
\hline ISI & $14.71 \pm 3.80$ & $13.82 \pm 4.09$ & 0.289 \\
\hline PSQI & $8.88 \pm 1.42$ & $8.03 \pm 1.45$ & $0.013^{*}$ \\
\hline ESS & $9.92 \pm 3.83$ & $7.79 \pm 4.64$ & $0.017^{*}$ \\
\hline \multicolumn{4}{|c|}{ Polysomnographic sleep parameters } \\
\hline oTST, min & $423.70 \pm 35.13$ & $376.69 \pm 54.64$ & $<0.001^{* * *}$ \\
\hline SE, \% & $88.66 \pm 7.48$ & $78.40 \pm 11.40$ & $<0.001^{* * *}$ \\
\hline oSOL, min & $11.14 \pm 14.91$ & $22.05 \pm 27.14$ & $0.002^{* *}$ \\
\hline REM Latency, min & $103.40 \pm 55.40$ & $142.67 \pm 70.77$ & $0.005^{* *}$ \\
\hline $\mathrm{N} 1, \%$ & $11.22 \pm 7.40$ & $17.16 \pm 12.18$ & $0.003^{* *}$ \\
\hline $\mathrm{N} 2, \%$ & $64.88 \pm 8.27$ & $61.33 \pm 9.93$ & 0.055 \\
\hline $\mathrm{N} 3, \%$ & $4.83 \pm 6.21$ & $3.17 \pm 5.02$ & 0.280 \\
\hline NREM, \% & $80.94 \pm 5.40$ & $81.67 \pm 6.15$ & 0.545 \\
\hline REM, \% & $19.06 \pm 5.40$ & $18.33 \pm 6.15$ & 0.544 \\
\hline WASO, min & $43.22 \pm 32.13$ & $81.74 \pm 52.41$ & $<0.001^{* * *}$ \\
\hline Total arousal, $\mathrm{N}$ & $109.31 \pm 50.20$ & $140.09 \pm 95.44$ & 0.180 \\
\hline AHI & $5.70 \pm 8.65$ & $10.18 \pm 16.99$ & 0.106 \\
\hline \multicolumn{4}{|l|}{ Psychological measures } \\
\hline CES-D & $12.17 \pm 9.36$ & $12.24 \pm 8.67$ & 0.798 \\
\hline BAI & $10.49 \pm 8.15$ & $7.58 \pm 6.62$ & 0.065 \\
\hline GARS & $24.25 \pm 12.24$ & $22.58 \pm 11.50$ & 0.704 \\
\hline SAPS & $30.93 \pm 6.13$ & $27.23 \pm 6.15$ & $0.007^{* *}$ \\
\hline
\end{tabular}

Data are presented as mean \pm standard deviation (SD). Independent t-tests for continuous variables and chi-square tests for categorical variables were used to determine the difference between the two groups. ${ }^{*} \mathrm{p}<0.05 ;{ }^{*} \mathrm{p}<0.01 ;{ }^{* * *} \mathrm{p}<0.001$. TST, total sleep time; SOL, sleep onset latency; ISI, insomnia severity index; PSQI, pittsburgh sleep quality index; ESS, Epworth Sleepiness Scale; SE, sleep efficiency; WASO, wake after sleep onset; AHI, apnea-hypopnea index; CES-D, Center for Epidemiologic Studies Depression Scale; BAI, Beck anxiety inventory; GARS, Global Assessment of Recent Stress Scale; SAPS, Smartphone Addiction Proneness Scale 
ney Test for continuous variables and chi-square tests or Fisher's exact test for categorical variables. Data are presented as mean \pm standard deviation for continuous variables and frequencies with percentages for categorical variables.

Univariable and multivariable logistic regression analyses were conducted to evaluate the psychological factors associated with sleep misperception. Odds ratios (ORs) and 95\% confidence intervals (CIs) were calculated. Demographic factors including age, gender, education years and factors that showed significance in the univariable logistic regression were selected for multivariable logistic regression model using stepwise backward selection. The data analyses were performed using SPSS version 22.0 (IBM Corp., Armonk, NY, USA), and $\mathrm{p}<0.05$ was considered statistically significant.

\section{RESULTS}

The demographic and clinical characteristics of the participants are shown in Table 1 . The mean age of the 105 participants was $40.61 \pm 11.62$ years. 49 (46.7\%) of them were female. Mean TST discrepancy of the participants was $-81.65 \pm 97.41$ minutes. Mean SOL discrepancy of the participants was 41.28 \pm 45.01 minutes.

\section{TST misperception}

Subjects were categorized into TST Misperception group and No TST Misperception group according to their TST discrepancy (Table 1). 65 (61.90\%) had underestimated their TSTs for more than 60 minutes and were categorized into the TST Misperception Group. 33 (31.43\%) subjects were categorized into the No TST Misperception group.
Age and gender distribution did not show any difference between the two groups (Table 2). Years of education were shorter in the TST Misperception group (14.74 \pm 2.16 vs. 15.91 $\pm 2.16, p=0.036$ ). While ISI scores were not significantly different between the two groups, TST Misperception Group had a significantly shorter subjective TST $(280.95 \pm 50.07$ vs. $366.06 \pm 56.73, \mathrm{p}<0.001)$ but a significantly longer objective TST ( $423.70 \pm 35.13$ vs. $376.69 \pm 54.64, \mathrm{p}<0.001)$. All the other objective sleep parameters including SE, SOL, WASO, and Total Arousal were significantly better in the TST Misperception group than the No TST Misperception Group. Also, the TST Misperception group had a lower percentage of N1 compared to the No TST Misperception Group (11.22 \pm 7.40 vs. $17.16 \pm 12.18, \mathrm{p}=0.003)$. Among the psychological measures, only scores of SAPS were significantly higher in the TST Misperception group (30.93 \pm 6.13 vs. $27.23 \pm 6.15, \mathrm{p}=0.007$ ).

Results of the logistic regression analyses are shown in Table 3. Multivariable logistic regression analyses revealed that higher scores of SAPS was an independent predictor of TST misperception (adjusted OR=1.14, 95\% CI=1.04-1.27, $\mathrm{p}=0.008$ ). Higher age (adjusted $\mathrm{OR}=1.07,95 \% \mathrm{CI}=1.01-1.13, \mathrm{p}=0.027$ ) and lower years of education (adjusted $\mathrm{OR}=0.69,95 \% \mathrm{CI}=$ $0.48-0.91, \mathrm{p}=0.017$ ) could also predict TST misperception.

\section{SOL misperception}

Subjects were categorized into SOL Misperception group and No SOL Misperception group according to their SOL discrepancy (Table 1). 55 (52.38\%) had overestimated their SOLs for more than 30 minutes and were categorized into the SOL Misperception Group. 49 (46.67\%) subjects were categorized into the No SOL Misperception group.

Table 3. Logistic regression analysis with TST misperception as a dependent variable

\begin{tabular}{|c|c|c|c|c|}
\hline \multirow{2}{*}{ Variables } & \multicolumn{2}{|c|}{ Univariable } & \multicolumn{2}{|c|}{ Multivariable } \\
\hline & OR (95\% CI) & $\mathrm{p}$ & OR (95\% CI) & $\mathrm{p}$ \\
\hline Age & $1.02(0.99-1.06)$ & 0.225 & $1.07(1.01-1.13)$ & $0.027^{*}$ \\
\hline Female & $1.17(0.5-2.72)$ & 0.716 & & \\
\hline Education & $0.76(0.59-0.95)$ & $0.023^{*}$ & $0.69(0.48-0.91)$ & $0.017^{*}$ \\
\hline ISI & $1.06(0.95-1.19)$ & 0.287 & & \\
\hline PSQI & $1.55(1.13-2.21)$ & $0.009^{* *}$ & $1.43(0.92-2.39)$ & 0.141 \\
\hline KESS & $1.14(1.02-1.28)$ & $0.02^{*}$ & $1.13(0.97-1.34)$ & 0.128 \\
\hline CES-D & $1(0.95-1.05)$ & 0.97 & & \\
\hline BAI & $1.06(1-1.14)$ & 0.084 & & \\
\hline GARS & $1.01(0.98-1.05)$ & 0.512 & & \\
\hline SAPS & $1.11(1.03-1.20)$ & $0.01^{*}$ & $1.14(1.04-1.27)$ & $0.008^{* *}$ \\
\hline
\end{tabular}

Multivariable logistic regression model using stepwise backward selection. ${ }^{*} \mathrm{p}<0.05 ;{ }^{* *} \mathrm{p}<0.01$. OR, odds ratio; CI, confidence interval; TST, total sleep time; ISI, insomnia severity index; PSQI, pittsburgh sleep quality index; ESS, Epworth Sleepiness Scale; CES-D, Center for Epidemiologic Studies Depression Scale; BAI, beck anxiety inventory; GARS, Global Assessment of Recent Stress Scale; SAPS, Smartphone Addiction Proneness Scale 
Demographic factors did not show any significant difference between the two groups (Table 4). While objective sleep parameters including TST and SOL did not show any significant difference between the two groups, subjective reports on sleep were worse in the SOL Misperception Group: subjective TST was shorter $(300.22 \pm 67.79$ vs. $336.73 \pm 71.25, \mathrm{p}=0.021)$, SOL was longer $(83.27 \pm 40.37$ vs. $28.63 \pm 17.63, \mathrm{p}<0.001)$, and scores of ISI were higher $(15.31 \pm 4.23$ vs. $13.43 \pm 4.07, \mathrm{p}=0.023)$ in the SOL Misperception Group. Among the psychological measures, only BAI was significantly higher in the SOL Misperception group (12.24 \pm 9.39 vs. $6.82 \pm 3.85, \mathrm{p}=0.001$ ).

Results of the logistic regression analyses are shown in Table 5. Multivariable logistic regression analyses revealed that higher scores of BAI was an independent predictor of SOL misper- ception (adjusted $\mathrm{OR}=1.16,95 \% \mathrm{CI}=1.05-1.31, \mathrm{p}=0.006$ ).

\section{DISCUSSION}

The present study examined the subjective-objective discrepancy of sleep duration and sleep latency in participants with insomnia disorder. The mean TST and SOL discrepancies (-81.65 \pm 97.41 minutes and 41.28 \pm 45.01 minutes, respectively) found in this study were within close range with the previous studies. ${ }^{28,34}$ Interestingly, objective polysomnographic results showed that those with TST misperception had a better quality of sleep than those without TST misperception, although subjective ISI scores did not show any difference between the two groups. On the contrary, those with or without

Table 4. Characteristics of the participants with or without SOL misperception

\begin{tabular}{|c|c|c|c|}
\hline Variables & SOL misperception $(\mathrm{N}=55)$ & No SOL misperception $(\mathrm{N}=49)$ & $\mathrm{p}$ \\
\hline Age, years & $40.09 \pm 12.93$ & $41.20 \pm 9.92$ & 0.710 \\
\hline Gender, Female & $31(56.36)$ & $24(48.98)$ & 0.578 \\
\hline Education, years & $14.90 \pm 2.19$ & $15.36 \pm 2.20$ & 0.312 \\
\hline \multicolumn{4}{|l|}{ Subjective sleep reports } \\
\hline sTST, min & $300.22 \pm 67.79$ & $336.73 \pm 71.25$ & $0.021^{*}$ \\
\hline sSOL, min & $83.27 \pm 40.37$ & $28.63 \pm 17.63$ & $<0.001^{* * *}$ \\
\hline ISI & $15.31 \pm 4.23$ & $13.43 \pm 4.07$ & $0.023^{*}$ \\
\hline PSQI & $8.84 \pm 1.36$ & $8.29 \pm 1.66$ & 0.080 \\
\hline KESS & $9.73 \pm 4.01$ & $8.47 \pm 4.47$ & 0.134 \\
\hline \multicolumn{4}{|c|}{ Polysomnographic sleep parameters } \\
\hline oTST, min & $399.76 \pm 64.56$ & $401.22 \pm 55.17$ & 0.948 \\
\hline $\mathrm{SE}, \%$ & $83.69 \pm 13.70$ & $83.60 \pm 11.60$ & 0.750 \\
\hline oSOL, min & $12.08 \pm 14.64$ & $16.00 \pm 21.71$ & 0.372 \\
\hline R Latency, min & $106.28 \pm 66.74$ & $135.52 \pm 70.71$ & $0.012^{*}$ \\
\hline $\mathrm{N} 1, \%$ & $12.32 \pm 7.51$ & $14.80 \pm 11.26$ & 0.460 \\
\hline $\mathrm{N} 2, \%$ & $63.72 \pm 8.74$ & $63.41 \pm 9.73$ & 0.990 \\
\hline $\mathrm{N} 3, \%$ & $5.16 \pm 6.33$ & $3.28 \pm 5.56$ & 0.157 \\
\hline NREM, \% & $81.20 \pm 5.03$ & $81.50 \pm 6.79$ & 0.803 \\
\hline REM, \% & $18.80 \pm 5.03$ & $18.50 \pm 6.79$ & 0.804 \\
\hline WASO, min & $66.17 \pm 62.91$ & $62.78 \pm 51.55$ & 0.920 \\
\hline Total Arousal, N & $113.44 \pm 51.73$ & $124.76 \pm 84.24$ & 0.822 \\
\hline AHI & $5.51 \pm 7.91$ & $9.15 \pm 15.17$ & 0.679 \\
\hline \multicolumn{4}{|l|}{ Psychological measures } \\
\hline CES-D & $14.13 \pm 10.91$ & $10.29 \pm 7.17$ & 0.097 \\
\hline BAI & $12.24 \pm 9.39$ & $6.82 \pm 3.85$ & $0.001^{* * *}$ \\
\hline GARS & $25.00 \pm 13.09$ & $22.53 \pm 11.34$ & 0.589 \\
\hline SAPS & $30.55 \pm 6.73$ & $28.70 \pm 5.45$ & 0.141 \\
\hline
\end{tabular}

Data are presented as mean \pm standard deviation (SD). Independent t-tests for continuous variables and chi-square tests for categorical variables were used to determine the difference between the two groups. ${ }^{*} \mathrm{p}<0.05$; ${ }^{* * *} \mathrm{p}<0.001$. TST, total sleep time; SOL, sleep onset latency; ISI, insomnia severity index; PSQI, pittsburgh sleep quality index; ESS, Epworth Sleepiness Scale; SE, sleep efficiency; WASO, wake after sleep onset; AHI, apnea-hypopnea index; CES-D, Center for Epidemiologic Studies Depression Scale; BAI, beck anxiety inventory; GARS, Global Assessment of Recent Stress Scale; SAPS, Smartphone Addiction Proneness Scale 
Table 5. Logistic regression analysis with SOL misperception as a dependent variable

\begin{tabular}{|c|c|c|c|c|}
\hline \multirow{2}{*}{ Variables } & \multicolumn{2}{|c|}{ Univariable } & \multicolumn{2}{|c|}{ Multivariable } \\
\hline & OR (95\% CI) & $\mathrm{p}$ & OR $(95 \% \mathrm{CI})$ & $\mathrm{p}$ \\
\hline Age & $0.99(0.96-1.03)$ & 0.623 & & \\
\hline Female & $1.35(0.62-2.93)$ & 0.452 & & \\
\hline Education & $0.91(0.75-1.09)$ & 0.308 & & \\
\hline $\operatorname{BMI}\left(\mathrm{kg} / \mathrm{m}^{2}\right)$ & $0.9(0.76-1.04)$ & 0.164 & & \\
\hline ISI & $1.12(1.02-1.24)$ & $0.027^{*}$ & $1.04(0.92-1.19)$ & 0.528 \\
\hline PSQI & $1.28(0.99-1.7)$ & 0.07 & $0.98(0.68-1.37)$ & 0.902 \\
\hline KESS & $1.07(0.98-1.18)$ & 0.135 & & \\
\hline CES-D & $1.05(1-1.1)$ & $0.045^{*}$ & $0.94(0.86-1.02)$ & 0.121 \\
\hline BAI & $1.11(1.04-1.19)$ & $0.002^{* *}$ & $1.16(1.05-1.31)$ & $0.006^{* *}$ \\
\hline GARS & $1.02(0.99-1.05)$ & 0.307 & & \\
\hline SAS & $1.05(0.98-1.13)$ & 0.142 & & \\
\hline
\end{tabular}

Multivariable logistic regression model using stepwise backward selection. ${ }^{*} \mathrm{p}<0.05$; ${ }^{* *} \mathrm{p}<0.01$. OR, odds ratio; CI, confidence interval; TST, total sleep time; ISI, insomnia severity index; PSQI, pittsburgh sleep quality index; ESS, Epworth Sleepiness Scale; CES-D, Center for Epidemiologic Studies Depression Scale; BAI, beck anxiety inventory; GARS, Global Assessment of Recent Stress Scale; SAPS, Smartphone Addiction Proneness Scale

SOL misperception did not show any difference in terms of objective sleep parameters, but subjective ISI scores were worse in those with SOL misperception. Regression analyses showed that higher smartphone addiction proneness, older age and shorter education years were significantly associated with TST Misperception. Anxiety was a significant predictor of SOL misperception.

To our knowledge, this is the first study to examine the association between smartphone addiction proneness and sleep discrepancy. Smartphone addiction has been reported to be associated with poorer sleep. ${ }^{35}$ Moreover, late night use of smartphone was significantly related with smartphone addiction. ${ }^{35}$ Smartphone-use before bed can increase the activity level of the nervous system, resulting in cognitive, emotional and physiological arousal. ${ }^{36,19}$ Particularly, cognitive arousal may maintain an increased level of sensory and memory processing during sleep, which reduces the ability to differentiate between sleep and wakefulness. ${ }^{15}$ Prior studies have demonstrated that greater cognitive arousal, especially pre-sleep cognitive activity contributes in greater TST discrepancy. ${ }^{13,37}$

It should be taken into consideration that subjects were not able to use the smartphone on the day of the polysomnography. Smartphone use before bed is an unstructured leisure activity which could easily be extended. Postponing sleep due to smartphone use is particularly associated with higher smartphone addiction proneness and longer smartphone time in bed. ${ }^{38}$ It has been reported that subjects with internet addiction had shorter total sleep duration as well as delayed bedtime than others. ${ }^{39}$ It is possible that participants' inability to use the smartphone on the night of the polysomnography may have resulted in actual longer sleep duration for those who usually use smartphone in bed for extended length of time. This could imply that subjects with higher smartphone addiction proneness have a distorted cognition that they have more severe sleep problems than they actually do.

The association between age and TST discrepancy found in this study is in line with previous studies which showed older adults have a tendency to perceive more sleep disturbances than those found in objective measures. ${ }^{40-42}$ Typically, aging is associated with physiological changes in sleep, such as increased fragmentation and arousals, which may play a role in sleep discrepancy. ${ }^{43,44}$ However, it should be noted that older adults above the age of 59 were excluded in this study.

TST discrepancy was also associated with shorter education years. No prior study has been conducted on the association between educational level and sleep discrepancy. Negative overgeneralization could have had played a role when subjects reported their habitual TST. ${ }^{37}$ We could hypothesize that individuals with shorter education are more vulnerable to overgeneralizing their experience of a few night experience of poor sleep and claim they have a habitual short duration of sleep.

The association between SOL discrepancy and anxiety was found in the present study. Hermans et al. ${ }^{34}$ demonstrated that sleep onset discrepancy is related to sleep fragmentation at the beginning of sleep. Subjects with insomnia may need longer continuous sleep, without being disturbed by sleep fragments, for the subject to differentiate sleep from wakefulness. Anxiety symptom have been reported to be associated with elevated sleep fragmentation. ${ }^{45}$ Also, greater sleep effort has been 
reported to be a significant predictor of SOL discrepancy. ${ }^{13}$ Reductions in sleep effort improved SOL discrepancy. ${ }^{46}$ This results may be related to the finding that high intention to fall asleep can worsen sleep quality, especially in terms of sleep fragmentation. ${ }^{47}$ Additionally, Gerlach et al. ${ }^{48}$ reported that pre-sleep worry is associated with insomnia-related interpretational bias. Excessive negative cognitive activity before sleep onset may trigger autonomic arousal, which has been suggested to be related to SOL discrepancy. ${ }^{49}$

There are several limitations that should be addressed. First, single night of polysomnography was used as an objective measure of sleep. Due to the lack of accommodation nights and repeated measurements over multiple nights, the objective measures of sleep may had been vulnerable to night-to-night variability and first-night effect. Second, the generalizability of the results may be limited by the subclinical nature of our participants. Participants were comprised of relatively mild to moderate insomnia patients probably due to the exclusion criteria which excluded those who had consumed any kind of sleep medication during the past month, as well as those with psychiatric disorders. Third, smartphone addiction proneness was relied upon self-reporting which could suffer from social desirability results. Also, amount of smartphone usage in bed, which could be particularly important, was not evaluated.

Being aware of the potential discrepancy between subjective perceptions and objective measures of sleep and wakefulness is important in assessing and treating insomnia. The present study provides empirical evidence to increase our understanding of the various factors that are associated with sleep discrepancy. Screening insomnia patients with smartphone addiction proneness may help predict the potential discrepancy between the patients' subjective reports and actual objective measures of sleep duration. Furthermore, behavioural intervention targeting problematic smartphone usage may decrease sleep discrepancy and distorted cognition on their sleep. Future prospective studies to determine causal directions on a clinical sample including patients with severe sleep problems are desirable. Additionally, more thorough evaluation on one's smartphone use should be considered.

\section{Availability of Data and Material}

The datasets generated or analyzed during the study are available from the corresponding author on reasonable request.

\section{Conflicts of Interest}

The authors have no potential conflicts of interest to disclose.

\section{Author Contributions}

Conceptualization: Han-Yong Jung. Data curation: Jeewon Lee. Formal analysis: Jeewon Lee. Funding acquisition: Shin-Gyeom Kim. Investigation: Jeewon Lee. Methodology: Soyoung Irene Lee. Software: HyunChul Youn. Supervision: Shin-Gyeom Kim. Witing-original draft: Jeewon Lee. Writing-review \& editing: Shin-Gyeom Kim.

\section{ORCID iDs}

Jeewon Lee

Han-Yong Jung

Soyoung Irene Lee

HyunChul Youn

Shin-Gyeom Kim

https://orcid.org/0000-0001-5930-1834 https://orcid.org/0000-0003-0089-0368 https://orcid.org/0000-0003-2473-2954 https://orcid.org/0000-0002-6557-5628 https://orcid.org/0000-0001-8196-655X

\section{Funding Statement}

This research was supported by High Value-added Food Technology Development Program, Ministry of Agriculture, Food and Rural Affairs, Republic of Korea (115044-03-3-HD030) and Soonchunhyang University Research Fund.

\section{REFERENCES}

1. Ohayon MM. Epidemiology of insomnia: what we know and what we still need to learn. Sleep Med Rev 2002;6:97-111.

2. Ohayon MM, Sagales T. Prevalence of insomnia and sleep characteristics in the general population of Spain. Sleep Med 2010;11:1010-1018.

3. Cao XL, Wang SB, Zhong BL, Zhang L, Ungvari GS, Ng CH, et al. The prevalence of insomnia in the general population in China: A metaanalysis. PLoS One 2017;12:e0170772.

4. Nowicki Z, Grabowski K, Cubała WJ, Nowicka-Sauer K, Zdrojewski T, Rutkowski M, et al. Prevalence of self-reported insomnia in general population of Poland. Psychiatr Pol 2016;50:165-173.

5. Chung S, Cho SW, Jo MW, Youn S, Lee J, Sim CS. The prevalence and incidence of insomnia in Korea during 2005 to 2013. Psychiatry Investig 2020;17:533-540.

6. Lee SY, Ju YJ, Lee JE, Kim YT, Hong SC, Choi YJ, et al. Factors associated with poor sleep quality in the Korean general population: providing information from the Korean version of the Pittsburgh Sleep Quality Index. J Affect Disord 2020;271:49-58.

7. American Psychiatric Association. Diagnostic and Statistical Manual of Mental Disorders (DSM- $\left.5^{\circledR}\right)$. Washington DC: American Psychiatric Pub; 2013.

8. Manconi M, Ferri R, Sagrada C, Punjabi NM, Tettamanzi E, Zucconi M, et al. Measuring the error in sleep estimation in normal subjects and in patients with insomnia. J Sleep Res 2010;19:478-486.

9. Rezaie L, Fobian AD, McCall WV, Khazaie H. Paradoxical insomnia and subjective-objective sleep discrepancy: a review. Sleep Med Rev 2018;40:196-202.

10. Harvey AG, Tang NK. (Mis) perception of sleep in insomnia: a puzzle and a resolution. Psychol Bull 2012;138:77-101.

11. Edinger JD, Krystal AD. Subtyping primary insomnia: is sleep state misperception a distinct clinical entity? Sleep Med Rev 2003;7:203-214.

12. Harvey AG. A cognitive model of insomnia. Behav Res Ther 2002;40: 869-893.

13. Herbert V, Pratt D, Emsley R, Kyle SD. Predictors of nightly subjectiveobjective sleep discrepancy in poor sleepers over a seven-day period. Brain Sci 2017;7:29.

14. Fernandez-Mendoza J, Calhoun SL, Bixler EO, Karataraki M, Liao D, Vela-Bueno A, et al. Sleep misperception and chronic insomnia in the general population: the role of objective sleep duration and psychological profiles. Psychosom Med 2011;73:88-97.

15. Tang NK, Harvey AG. Effects of cognitive arousal and physiological arousal on sleep perception. Sleep 2004; 27:69-78.

16. Lepp A, Li J, Barkley JE, Salehi-Esfahani S. Exploring the relationships between college students' cell phone use, personality and leisure. Comput Human Behav 2015;43:210-219.

17. Exelmans L, Van den Bulck J. Bedtime mobile phone use and sleep in adults. Soc Sci Med 2016;148:93-101.

18. Fossum IN, Nordnes LT, Storemark SS, Bjorvatn B, Pallesen S. The association between use of electronic media in bed before going to sleep and insomnia symptoms, daytime sleepiness, morningness, and chro- 
notype. Behav Sleep Med 2014;12:343-357.

19. Pieters D, De Valck E, Vandekerckhove M, Pirrera S, Wuyts J, Exadaktylos V, et al. Effects of pre-sleep media use on sleep/wake patterns and daytime functioning among adolescents: the moderating role of parental control. Behav Sleep Med 2014;12:427-443.

20. Lee J, Jung HY, Lee SI, Choi JH, Kim SG. Effects of Passiflora incarnata Linnaeus on polysomnographic sleep parameters in subjects with insomnia disorder: a double-blind randomized placebo-controlled study. Int Clin Psychopharmacol 2020;35:29-35.

21. Cho YW, Song ML, Morin CM. Validation of a Korean version of the insomnia severity index. J Clin Neurol 2014;10:210-215.

22. Sohn SI, Kim DH, Lee MY, Cho YW. The reliability and validity of the Korean version of the Pittsburgh Sleep Quality Index. Sleep Breath 2012; 16:803-812

23. Cho YW, Lee JH, Son HK, Lee SH, Shin C, Johns MW. The reliability and validity of the Korean version of the Epworth sleepiness scale. Sleep Breath 2011;15:377-384.

24. Shin K, Kim D, Jung Y. Development of Korean Smart Phone Addiction Proneness Scale for Youth and Adults. Seoul: Korean National Information Society Agency; 2011.

25. Radloff LS. The CES-D scale: A self-report depression scale for research in the general population. Appl Psychol Meas 1977;1:385-401.

26. Fydrich T, Dowdall D, Chambless DL. Reliability and validity of the Beck Anxiety Inventory. J Anxiety Disord 1992;6:55-61.

27. Linn MW. A global assessment of recent stress (GARS) scale. Int J Psychiatr Med 1986;15:47-59.

28. Bianchi MT, Williams KL, Mckinney S, Ellenbogen JM. The subjectiveobjective mismatch in sleep perception among those with insomnia and sleep apnea. J Sleep Res 2013;22:557-568.

29. Liao J, Zhu S, Li X. Anxiety and depression in paradoxical insomnia: a case-control study. Neuropsychiatr Dis Treat 2018;14:231-238.

30. Normand MP, St-Hilaire P, Bastien CH. Sleep spindles characteristics in insomnia sufferers and their relationship with sleep misperception. Neural Plast 2016;2016:6413473.

31. St-Jean G, Turcotte I, Pérusse AD, Bastien CH. REM and NREM power spectral analysis on two consecutive nights in psychophysiological and paradoxical insomnia sufferers. Int J Psychophysiol 2013;89:181-194.

32. Salin-Pascual RJ, Roehrs TA, Merlotti LA, Zorick F, Roth T. Long-term study of the sleep of insomnia patients with sleep state misperception and other insomnia patients. Am J Psychiatry 1992;149:904-908.

33. Castelnovo A, Ferri R, Punjabi NM, Castronovo V, Garbazza C, Zucconi M, et al. The paradox of paradoxical insomnia: a theoretical review towards a unifying evidence-based definition. Sleep Med Rev 2019;44: 70-82.

34. Hermans LW, Leufkens TR, van Gilst MM, Weysen T, Ross M, Anderer P, et al. Sleep EEG characteristics associated with sleep onset misper- ception. Sleep Med 2019;57:70-79.

35. Sohn SY, Krasnoff L, Rees P, Kalk NJ, Carter B. The association between smartphone addiction and sleep: a UK cross-sectional study of young adults. Front Psychiatry 2021;12:629407.

36. Ivarsson M, Anderson M, Åkerstedt T, Lindblad F. Playing a violent television game affects heart rate variability. Acta Paediatr 2009;98:166-172.

37. Takano K, Boddez Y, Raes F. I sleep with my Mind's eye open: Cognitive arousal and overgeneralization underpin the misperception of sleep. J Behav Ther Exp Psychiatry 2016;52:157-165.

38. Paik SH, Park CH, Kim JY, Chun JW, Choi JS, Kim DJ. Prolonged bedtime smartphone use is associated with altered resting-state functional connectivity of the insula in adult smartphone users. Front Psychiatry 2019;10:516.

39. Kawabe K, Horiuchi F, Oka Y, Ueno SI. Association between sleep habits and problems and internet addiction in adolescents. Psychiatry Investig 2019;16:581-587.

40. Tsuchiyama K, Nagayama H, Kudo K, Kojima K, Yamada K. Discrepancy between subjective and objective sleep in patients with depression. Psychiatr Clin Neurosci 2003;57:259-264.

41. Kay DB, Buysse DJ, Germain A, Hall M, Monk TH. Subjective-objective sleep discrepancy among older adults: associations with insomnia diagnosis and insomnia treatment. J Sleep Res 2015;24:32-39.

42. Williams JM, Kay DB, Rowe M, McCrae CS. Sleep discrepancy, sleep complaint, and poor sleep among older adults. J Gerontol B Psychol Sci Soc Sci 2013;68:712-720.

43. Edwards BA, O’Driscoll DM, Ali A, Jordan AS, Trinder J, Malhotra A. Aging and sleep: physiology and pathophysiology. Seminars in respiratory and critical care medicine. NIH Public Access 2010;618.

44. Parrino L, Milioli G, De Paolis F, Grassi A, Terzano MG. Paradoxical insomnia: the role of CAP and arousals in sleep misperception. Sleep Med 2009;10:1139-1145.

45. Spira AP, Stone K, Beaudreau SA, Ancoli-Israel S, Yaffe K. Anxiety symptoms and objectively measured sleep quality in older women. Am J Geriatr Psychiatry 2009;17:136-143.

46. Broomfield N. Initial Insomnia and Paradoxical Intention: An Experimental Investigation of Putative Mechanisms Using Subjective and Actigraphic Measurement of Sleep. Cambridge: ProQuest Dissertations \& Theses; 2001.

47. Rasskazova E, Zavalko I, Tkhostov A, Dorohov V. High intention to fall asleep causes sleep fragmentation. J Sleep Res 2014;23:297-303.

48. Gerlach F, Ehring T, Werner GG, Takano K. Insomnia-related interpretational bias is associated with pre-sleep worry. J Sleep Res 2020;29:e12938.

49. Maes J, Verbraecken J, Willemen M, De Volder I, Van Gastel A, Michiels $\mathrm{N}$, et al. Sleep misperception, EEG characteristics and autonomic nervous system activity in primary insomnia: a retrospective study on polysomnographic data. Int J Psychophysiol 2014;91:163-171. 